\title{
Gfi-I promotes proliferation of human cervical carcinoma via targeting of FBW7 ubiquitin ligase expression
}

This article was published in the following Dove Press journal: Cancer Management and Research

\author{
Hongbing Cai ${ }^{1-3}$ \\ Fan Zhang ${ }^{1-3}$ \\ Zhen $\mathrm{Li}^{1-3}$ \\ 'Department of Gynecological \\ Oncology, Zhongnan Hospital of \\ Wuhan University, ${ }^{2}$ Hubei Clinical \\ Cancer Study Center, ${ }^{3}$ Hubei Key \\ Laboratory of Tumor Biological \\ Behaviors, Wuhan, People's Republic \\ of China
}

Background: The independent growth factor 1 (Gfi-1) is a transcription factor essential for several diverse hematopoietic functions and developments. However, the role and molecular mechanism of Gfi-1 in the development and progression of cervical cancer remains unclear.

Purpose: The present study investigates the relation of expression of Gfi-1 with prognoses in patients with cervical cancer.

Methods: We used Western blot and reverse transcription polymerase chain reaction (RT-PCR) and the inhibition of proliferation and metastasis of cervical cancer cells in vitro.

Results: This study confirms that the expression of Gfi-1 in cervical cancer tissues was higher than that in adjacent normal tissues. The level of Gfi-1 mRNA in human cervical cancer tissues was significantly higher than that in normal tissues adjacent to cancer. Furthermore, overexpression of Gfi-1 promoted cell proliferation, colony formation, and migration of cervical cancer cells. The increased expression of Gfi-1 promotes the proliferation of cervical cancer cells targeting the tumor suppressor F-box and WD repeat domain containing 7 (FBW7). Clinically, our data suggest that overexpression of Gfi-1 is associated with poor prognosis in patients with cervical cancer. In a tumor xenograft model, knockdown of Gfi-1 inhibited the tumor growth of Hela cells in vivo. Conclusion: Our results reveal that Gfi-1 plays an important role in cervical cancer and Gfi-1/ FBW7 axis serves as a potential therapeutic target for cervical cancer.

Keywords: cervical cancer, Gfi-1, proliferation, FBW7

\section{Introduction}

Cervical cancer is one of the leading causes of cancer deaths in women worldwide. Approximately 500,000 new cases of cervical cancer have been estimated and 280,000 people die each year. ${ }^{1}$ More than $80 \%$ of cervical cancer patients and 150,000 new cases of cervical cancer in developing countries ${ }^{2}$ account for $\sim 150,000$ of China's annual cases and $\sim 30 \%$ of the world's new cases. Over the past few decades, although overall incident or mortality rates have declined steadily among cervical cancer patients globally, these rates have risen significantly for patients in developing countries, including China. ${ }^{3-5}$ Moreover, the estimated 5-year survival rates decreased dramatically if women were diagnosed with advanced-stage cervical cancer. ${ }^{5}$ The determination of tumor markers plays an important role in the early diagnosis and treatment of cervical precancerous lesions and cervical cancer, which can effectively reduce the occurrence of cervical cancer and prolong the survival time of patients with cervical cancer. ${ }^{6}$

Gfi-1 is a transcription inhibitor of DNA binding. The N-terminal Snail/Gfi-1 domain is necessary for nuclear localization. In contrast, the C-terminal zinc finger
Correspondence: Zhen Li

Department of Gynecological Oncology, Zhongnan Hospital of Wuhan University, No 169 East Lake Road, Wuhan 43007I, People's Republic of China

Tel +86I587l40 48I8

Email lizhen02@yeah.net 
domain is required for binding DNA elements to a sequence of 5'-TAAATCAC(A/T)GCA-3'. ${ }^{7}$ Gfi- 1 can be detected in hepatic stellate cells (HSCs), common lymphatic progenitor cells, and granule/monocyte progenitor cells, but it does not exist in common bone marrow progenitor cells and megakaryocyte/erythrocyte progenitor cells. ${ }^{8}$ Studies have shown that Gfi-1 knockout mice bone marrow B- and T-cell lineages and HSCs defects indicate that Gfi-1 plays a key role in different cell lineage. ${ }^{9}$ Gfi-1, a cooperating oncogene in lymphoid cells, ${ }^{10,11}$ unexpectedly restricts the proliferation of HSCs. Meanwhile, Gfi-1 was rapidly downregulated upon the induction of eosinophilic differentiation in late neutrophilic differentiation, but the negative effect was eosinophilic development. ${ }^{12}$ Gfi-1 is an essential regulator of the ectonucleotidase expression of Th17 cells during differentiation. Th17 cells express nucleotide enzymes that inhibit the function of T cells, thereby inhibiting antitumor immunity. ${ }^{13}$ Inhibiting Gfi-1 can not only cure acute lymphoblastic leukemia (ALL) in mice but also hinder the expansion of human primary cells. ${ }^{14}$ Thus, Gfi-1 as a so-called "oncorequisite" factor does not play a role in the process of malignant transformation but is critically required by tumor cells for survival and progress. ${ }^{14}$ Gfi-1 suppressed T-cell death by inhibiting the activation and rewriting of proapoptotic factors in G1 cell cycle checkpoints. Gfi-1 regulates the proliferation of IL-4/ STAT6-dependent Th2 cells and IL-6/STAT3-mediated proliferation of antigenic stimulation. ${ }^{15}$ Several targets of Gfi-1, including STAT 5B, Mcl-1, Runx2, and SOCS1, have been found in many types of cells. ${ }^{16}$ These results suggest that more targets of Gfi-1 remain, encouraging further exploration to obtain deeper insights into the function of Gfi-1. However, in the occurrence and development of tumors, the definition of the mechanism of Gfi-1 expression abnormality and its regulation is still unclear; in addition, the link between Gfi-1 and cervical cancer has not been reported.

$\mathrm{F}$ boxes and WD repeat domains containing 7(FBW7) is apart of the substrate recognition of Skp1 Cul1 F box(SCF) that degradation of ubiquitin ligase complexes, as well as a variety of tumor suppressor proteins, including cyclin $\mathrm{E}$, Notch, c-myc and c-jun, is involved in inhibiting the development of cancer. ${ }^{17-19}$ A recent study showed that FBXW7 is downregulated in cervical cancer and associated with risk factors of cervical cancer, such as poor grade, lymphovascular space invasion, and lymph node metastasis. ${ }^{20}$ However, studies on the regulation of FBW7 upstream signaling pathways are very limited. In this study, we found that the expression of Gfi-1 was increased in cervical cancer tissues, which contributed to the adverse prognosis of the clinical patients.
In addition, these findings suggest that the Gfi-1/FBW7 axis is associated with a malignant phenotype and is a potential therapeutic target for cervical cancer.

\section{Materials and methods Cell culture}

Human embryonic kidney 293T cells and human cervical carcinoma cell lines HeLa and SiHa were purchased from the American Type Culture Collection (ATCC). All cell lines were cultured in Dulbecco's modified eaglemedium (DMEM) (Thermo Fisher Scientific, Waltham, MA, USA), supplemented with $10 \%$ fetal bovine serum (Hyclone, Logan, USA), penicillin-streptomycin (100 U/mL; Hyclone), and glutamine ( $2 \mathrm{mM}$; Hyclone). Cell cultures were maintained in a humidified atmosphere of $5 \% \mathrm{CO}_{2}$ at $37^{\circ} \mathrm{C}$. The cells were dissociated with $0.25 \%$ trypsin and $0.02 \%$ EDTA solution and subcultured once in every $2-3$ days.

\section{Specimens}

During 2013-2016, the cervical cancer tissue of 200 patients was collected in inpatient and outpatient operations at Zhongnan Hospital of Wuhan University. According to the International Federation of Gynecology and Obstetrics (FIGO) staging standards (2009) and histopathological classification, cervical cancer clinical staging, all patients provided complete clinical data and had not received chemotherapy, radiotherapy, biological treatment, or surgical treatment. After reviewing, the Department of Pathology of Wuhan University made a pathological diagnosis of each sample. The age difference of the three groups was not statistically significant.

\section{Cell viability assay}

In a 96 -well plate, $2 \times 10^{3}$ cells were cultured and $20 \mu \mathrm{L}$ of MTT solution was added at the specified time point and incubated for $4 \mathrm{~h}$. The culture medium $(200 \mu \mathrm{L}$ of DMEM containing $10 \%$ fetal bovine serum) was replaced by $150 \mu \mathrm{L}$ of DMSO, and the plate was vibrated for $10 \mathrm{~min}$, and absorbance measured at $490 \mathrm{~nm}$ to determine the number of living cells in each well. All experiments were performed three times.

\section{Colony formation assay}

Cells were seeded in six-well plates at a density of $1 \times 10^{3}$ cells/well and allowed to attach to the plate overnight prior to treatment. Cells were incubated for 10 days. Then, they were fixed with $4 \%$ formaldehyde and stained with crystal violet. The number of colonies with at least 50 cells was counted under a microscope at $4 \times$ magnification. 


\section{Lentivirus production and transduction of target cells}

We constructed the lentiviruses vectors of overexpression Gfi-1. Gfi-1 shRNA lentivirus (TRCN0000020468; Open Biosystems, Huntsville, AL, USA) was used to infect cells in the presence of Polybrene. Forty-eight hours later, Hela and $\mathrm{SiHa}$ cells were cultured in medium containing puromycin for the selection of stable clones. The FBW7 expression lentivirus was purchased from Shanghai GeneChem Co (Shanghai, China).

\section{Coimmunoprecipitation and Western blotting}

The HEK-293T cells cultured in $10 \mathrm{~cm}$ Petri dishes were transfected with calcium phosphate precipitation method. After $36 \mathrm{~h}$, the transfected cells were lysed with radioimmunoprecipitation assay (RIPA) buffer containing the complete protease inhibitor (Roche diagnosis method). The protein with the marked antibody and protein $\mathrm{A} / \mathrm{G}$ beads was incubated, in a mild rotation for $4 \mathrm{~h}$. Beads were washed five times with RIPA. The binding protein was eluted in buffer at $100^{\circ} \mathrm{C}$ for $5 \mathrm{~min}$. Immunoprecipitation and protein lysis were separated by sodium dodecyl sulfate-polyacrylamide gel electrophoresis, transferred to polyvinylidene fluoride (PVDF) membranes (Bio-Rad Laboratories Inc., Hercules, CA, USA), and blocked with $0.5 \%$ BSA (Sigma-Aldrich Co., St Louis, MO, USA). Blots were probed with antibodies to Gfi-1 (N20; Santa Cruz Biotechnology Inc., Dallas, TX, USA), Flag (M2; Sigma-Aldrich Co.), actin (AC40; Sigma-Aldrich Co.), and hemagglutinin (SC-57592; Santa Cruz Biotechnology Inc.)

\section{Transwell assays}

The transwell assay was performed in 24-well plates. For the invasion assay, the top side of a polycarbonate filter was coated with Matrigel and placed in the upper chamber of a BioCoat Invasion Chamber (BD, Franklin Lakes, NJ, USA). Cells were resuspended in serum-free medium $\left(2 \times 10^{4} / 200 \mu \mathrm{L}\right)$, added to the top well, and incubated at $37^{\circ} \mathrm{C}$ for $24 \mathrm{~h}$. Cells that crossed to the underside of the transwell membrane were fixed in $4 \%$ formaldehyde, then stained with $0.1 \%$ crystal violet, and counted under a microscope.

\section{RNA extraction and reverse transcription polymerase chain reaction (RT-PCR)}

Total RNA was extracted using the TRIzol Reagent (SigmaAldrich Co.) according to the manufacturer's protocol. RNA was then treated with DNaseI (Hoffman-La Roche Ltd., Basel,
Switzerland), purified by an Rneasy column (Qiagen NV, Venlo, the Netherlands), and electrophoresed in the $5^{\prime}$ - rapid amplification of cDNA ends experiment to determine the integrity of the RNA. The complementary DNA (cDNA) was synthesized from $1 \mu \mathrm{g}$ of total RNA using the random hexapod (progo) and superscript III reverse transcriptase (Thermo Fisher Scientific). RTPCR was carried out on a panel of cell lines and tumor samples. The qPCR primers are as follows: $5^{\prime}$-GCACCGTCAAGGCTGAGAAC- ${ }^{\prime}$ and $5^{\prime}$-TGGTGAAGACGCCAGTGGA-3' for GAPDH; 5'-AAAGAGTTGTTAGCGGTTCTCG-3' and 5'-CCACATGGATACCATCAAACTG-3' for FBW7.

\section{Cell cycle analysis}

In the cell cycle analysis, the $4 \times 10^{5}$ cells were synchronized by serum starvation for $24 \mathrm{~h}$, and then re-stimulated by the addition of $10 \%$ of fetal bovine serum to re-enter the cell cycle for $9 \mathrm{~h}$. The cells were harvested and fixed in $75 \%$ of ethanol and kept overnight at $4^{\circ} \mathrm{C}$. Cells were incubated with RNase A at $37^{\circ} \mathrm{C}$ for $30 \mathrm{~min}$ and then stained with propidium iodide (PI) at $37^{\circ} \mathrm{C}$ for $30 \mathrm{~min}$. Cell cycle was measured using flow cytometry.

\section{Tumor xenograft}

Nude mice (BALB/c; specific pathogen-free grade; 6 weeks old, subcutaneous injection of $200 \mu \mathrm{L}\left[5 \times 10^{6}\right]$ cells) were used in this study. The tumor size was measured with a vernier caliper every 3 days. Tumor volumes were determined according to the following formula: $A \times B^{2} / 2$, where $A$ is the largest diameter and $B$ is the perpendicular diameter. On the 30th day after the injection, the mice were sacrificed, and the tumor was withdrawn. All operations involving live mice were approved by the Animal Care and Use Committee of Wuhan University.

\section{Statistical analyses}

Means were compared using the unpaired two-tailed Student's $t$-test. A $P$-value of $<0.05$ was considered statistically significant in all calculations.

\section{Ethics approval and consent to participate}

All animals were fed and used in accordance with the guidelines of the Institutional Animal Care and Use Committee at Zhongnan Hospital of Wuhan University. Ethical approval for the human tissue study was obtained from Wuhan university. Written informed consent was obtained from all participants before beginning the study. 


\section{Results}

\section{Association between Gfi-I expression} and clinicopathological characteristics

To further explore the role of Gfi-1 in cervical cancer, we conducted real-time PCR for 200 cervical cancer tissues and adjacent normal tissues in the training cohort. The Gfi-1 expression was significantly increased compared with adjacent normal tissues (Figure 1A). These data showed that increased expression of Gfi-1 may be a common event in cervical cancer. For clinical stage, the expression of Gfi-1 in III and IV cancer tissues was significantly higher than that in I and II cancer tissues (Figure $1 \mathrm{~B})(P<0.01)$. To study the relationship between Gfi-1 expression and clinicopathological features, we analyzed its correlation in the training cohort. As shown in Table 1, the expression of Gf-1 was closely related to the degree of differentiation in cervical cancer $(P=0.052)$, clinical stage $(P<0.001)$, diameter of tumor $(P<0.001)$, metastasis of lymph nodes $(P<0.05)$ and vascular invasion $(P<0.05)$; however, there were no significant differences between Gfi-1 expression and age, human papillomavirus infection in patients with cervical carcinoma. Furthermore, patients were divided into the following two groups: high Gfi-1 expression $(n=113)$ and low Gfi-1 expression $(n=87)$ groups. There was a striking negative correlation between Gfi-1 expression intensity and overall survival (OS; $P<0.01$ ) (Figure 1C). Western blot was conducted to investigate Gfi-1 at a protein level (Figure 1D). The results revealed low Gfi-1 expression in normal cervical tissues but high Gfi-1 expression in cervical squamous carcinoma tissues $(P<0.001)$.

\section{Gfi-I promotes proliferation of human cervical cancer cells}

To investigate the roles of Gfi-1 in cervical cancer cells, Gfi-1 overexpression Hela and SiHa cells were generated and the efficiency of Gfi-1 overexpression was determined by qRT-PCR assay (Figure 2A). Then, we performed the MTT assay to evaluate cell proliferation. Overexpression of Gfi-1 significantly increased cell proliferation in Hela and SiHa cells (Figure 2B). Moreover, the effect of Gfi-1 on the proliferation of cervical carcinoma cells was examined
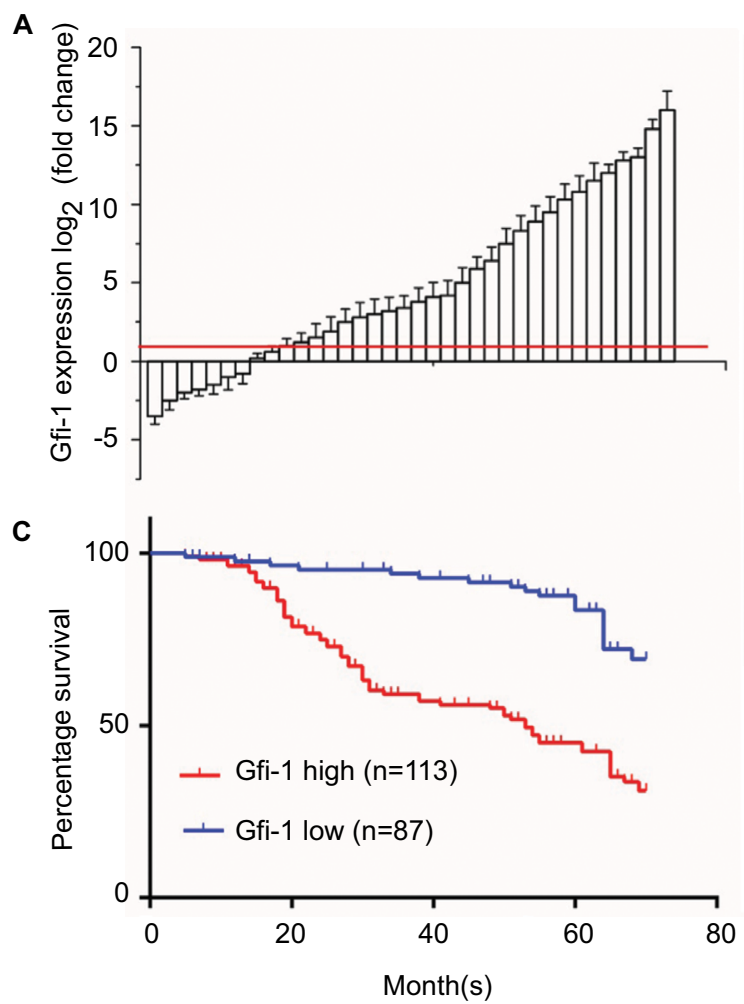

B
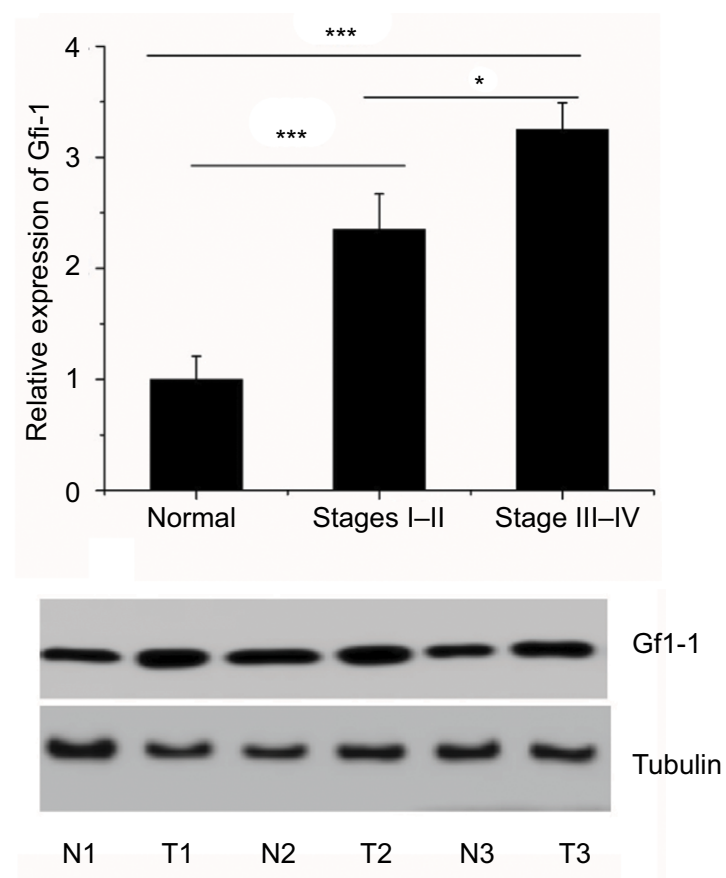

Figure I Association between Gfi-I expression and clinicopathological characteristics.

Notes: (A) Gfi-I expression in 200 cervical cancer tissues and adjacent normal tissues. Determination of Gfi-I expression levels of b-actin by fluorescent quantitative PCR and b-actin standard. The red line indicates fold change of Gfi-I equal to 2. (B) A total of 200 patients were divided into stages I, II, and III and IV. The graph shows the expression of $\mathrm{Gfi}-\mathrm{I}$ in each group. ${ }^{*} P<0.05$, ${ }^{* * * P}<0.00 \mathrm{I}$. (C) The OS rates of the 200 cervical cancer patients were compared with the Gfi-I low and Gfi-I high groups. (D) Gfi-I protein levels were detected in tissues of normal or cervical cancer patients by Western blotting. N, normal cervical tissue. Numbers I-6 indicate patient number. Statistical significance was determined using the log-rank test.

Abbreviations: OS, overall survival; PCR, polymerase chain reaction; T, tumor cervical tissue. 
Table I

\begin{tabular}{|c|c|c|c|}
\hline Clinicopathological feature & $\mathbf{n}$ & Gfi-I expression & $\mathbf{P}$ \\
\hline \multicolumn{4}{|l|}{ Age (years) } \\
\hline$>40$ & 162 & $2.45 \pm 0.318$ & 0.635 \\
\hline$\leq 40$ & 38 & $2.505 \pm 0.032$ & \\
\hline \multicolumn{4}{|l|}{ Degree of differentiation } \\
\hline Poor & 50 & $3.336 \pm 0.232$ & 0.052 \\
\hline Middle & 113 & $2.285 \pm 0.217$ & \\
\hline High & 37 & $1.935 \pm 0.205$ & \\
\hline \multicolumn{4}{|l|}{ Clinical stage } \\
\hline I & 87 & $1.785 \pm 0.028$ & $<0.001$ \\
\hline II & 78 & $2.913 \pm 0.103$ & \\
\hline III & 35 & $3.339 \pm 0.215$ & \\
\hline \multicolumn{4}{|l|}{ Tumor diameter $(\mathrm{cm})$} \\
\hline$<4$ & 148 & $2.236 \pm 0.234$ & $<0.001$ \\
\hline$\geq 4$ & 52 & $3.216 \pm 0.185$ & \\
\hline \multicolumn{4}{|l|}{ Lymph-node metastases } \\
\hline Yes & 63 & $2.932 \pm 0.131$ & 0.034 \\
\hline No & 137 & $2.234 \pm 0.118$ & \\
\hline \multicolumn{4}{|l|}{ HPV infection } \\
\hline Positive & 145 & $2.482 \pm 0.234$ & 0.964 \\
\hline Negative & 55 & $2.506 \pm 0.164$ & \\
\hline \multicolumn{4}{|l|}{ Vascular invasion } \\
\hline Positive & 87 & $2.891 \pm 0.285$ & 0.046 \\
\hline Negative & 113 & $2.215 \pm 0.312$ & \\
\hline
\end{tabular}

Abbreviation: HPV, human papillomavirus.

in a colony formation assay. We found that after 10 days of incubation with overexpression of Gfi-1, colony formation was significantly increased in $\mathrm{HeLa}$ and $\mathrm{SiHa}$ cells compared to the control group (Figure 2C). To determine Gfi-1 effects on cervical carcinoma cell migration and invasion ability, we performed wound healing and cell invasion assays. As expected, overexpression of Gfi- 1 significantly promoted the migration of $\mathrm{HeLa}$ and $\mathrm{SiHa}$ cells in vitro $(P<0.05)$ (Figure 2D). Since the increase in Gfi-1 results in an increased proliferation of cervical cells, cell cycle analysis is used to detect whether overexpression of Gfi-1 promotes cell cycle transitions at specific stages of the cell cycle. The results showed no difference in the percentage of overexpression of Gfi-1 cells in G1 phase, a significant decrease in S phase cells, and a significant increase in G2/M cells (Figure 2E). These results indicated that Gfi-1 effectively enhanced the viability of cervical carcinoma cells by promoting $\mathrm{G} 2 / \mathrm{M}$ cell cycle transition.

\section{Gfi-I target of FBW7 exerts its function by suppressing FBW7 expression}

Because FBW7 functions as a ubiquitin ligase, Gfi- 1 is involved in the ubiquitination and degradation of $26 \mathrm{~S}$ proteasome in myeloid cells. ${ }^{21}$ We studied whether FBW7 has a direct relationship with Gfi-1.
To this end, we transfected the HEK-293T cells into Flag-Gfi-1, with or without HA-FBW7. In the Western blot analysis, HA staining showed protein binding to FBW7 after immunoprecipitation (Figure 3A). This result indicated that FBW7 has a direct relationship with Gfi-1. Gfi-1 upregulation in cervical cancer of Hela cells and $\mathrm{SiHa}$ that reduce the mRNA expression of FBW7 (Figure 3B). In addition, the expression of Gfi-1 in human cervical cancer tissue was negatively correlated with mRNA expression level (Figure 3C). It has been shown that the key oncogenic FBW7 substrates include c-Myc, Mcl-1, and cyclin E. To better understand the mechanism of Gfi-1 involved in the proliferation of cervical cancer cells, the changes in Gfi-1-induced signal transduction pathway in SiHa and Hela cells were detected by Western blot. Interestingly, as well as Gfi-1, the FBW7-shRNA inhibits the expression of c-Myc and cyclin E, at protein levels, in Hela and SiHa cells (Figure 3D and E). Next, we studied the proliferation of Hela-Gfi-1 and SiHa-Gfi-1 and the expression of FBW7 gene and found that the expression of FBW7 was increased enough to cause the proliferation inhibition of cervical cancer cells (Figure 3F and G).

\section{FBW7 blocks the Gfi-I-induced proliferation of Hela cells in vivo}

To further explore the influence of Gfi-1 on cervical cell growth, we investigated the effects of Gfi- 1 on the xenograft model. As evaluated by tumor volumes, knockdown of Gfi-1 substantially decreased Hela cells' growth (Figure 4A and B). In addition, Hela knockdown of Gfi-1 led to a significant decrease in tumor weight compared with the control cells (Figure 4C). In addition, the expression of FBW7 can be increased by injecting FBW7 overexpression Hela cells and blocking the proliferation of Gfi-1 induced in xenograft models. Moreover, increased FBW7 expression was sufficient to block the Gfi-1-induced proliferation in the xenograft model by injecting FBW7 overexpression Hela cells (Figure 4D-F). These data demonstrate that Gfi-1 promotes cervical cancer tumorigenesis and FBW7 blocks the Gfi-1-induced proliferation on tumor growth in vivo.

\section{Discussion}

Cervical cancer is a malignant tumor of cervical epithelium, which is a leading gynecologic malignancy in China. The invasion and metastasis of malignant cells are mainly caused by uncontrolled proliferation of cells. Molecular biology studies show that the uncontrolled proliferation of cells is related to the abnormal expression of proto-oncogenes and tumor suppressor genes. Inactivation and mutation of tumor 
A
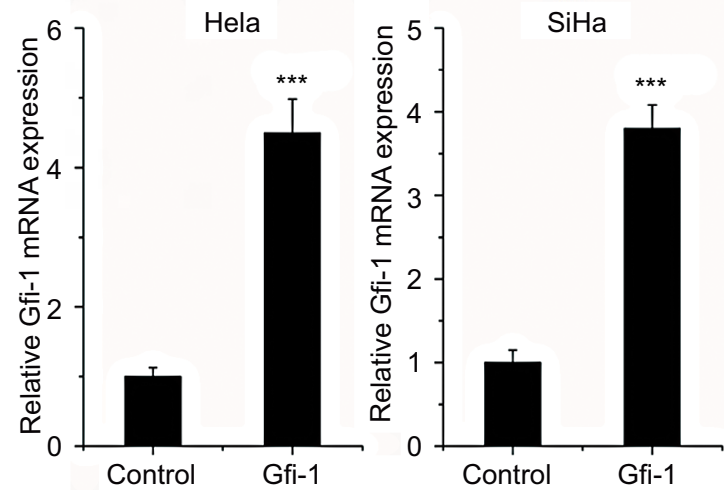

C
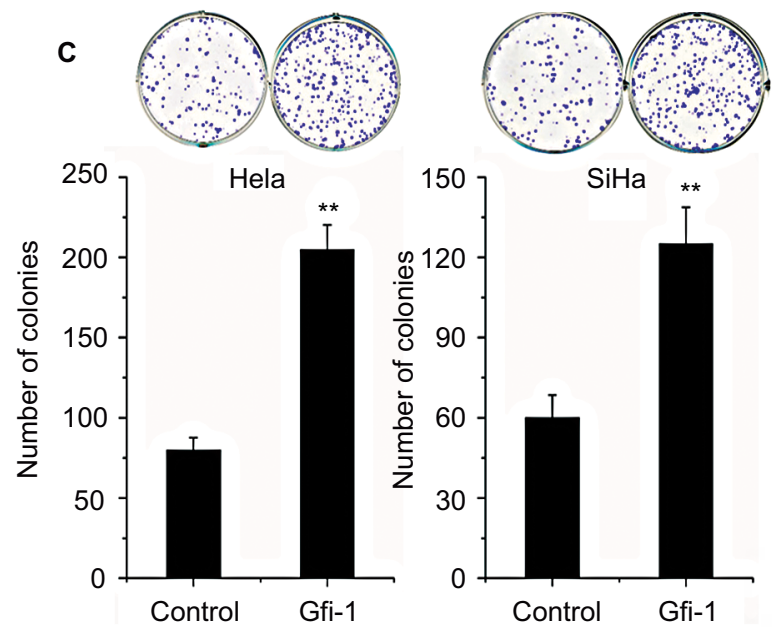

B
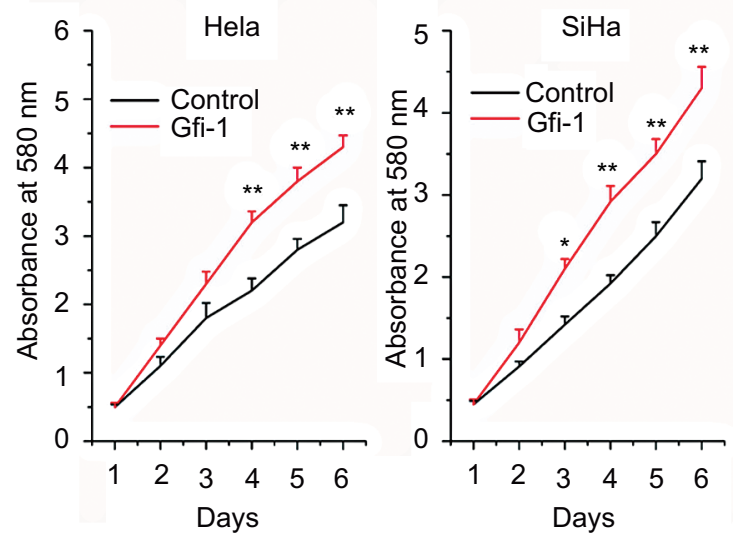

D

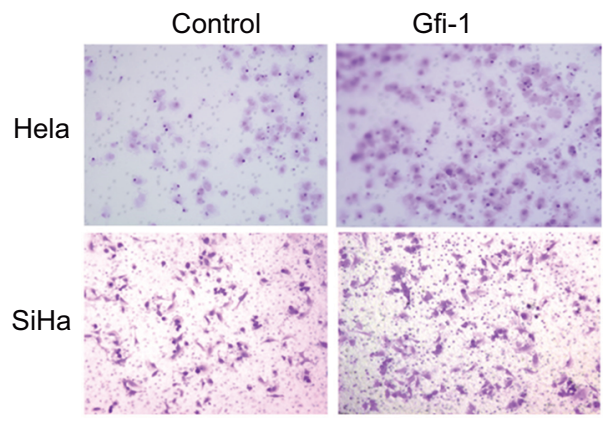

E

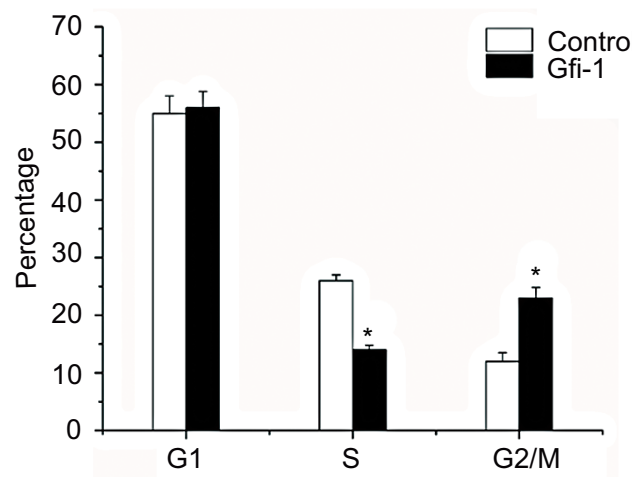

Figure 2 Gfi-I promotes proliferation and metastasis of human cervical cancer cells.

Notes: (A) The cells were transfected with Gfi-I and then used for real-time analysis. (B) MTT assay was used to determine the cell proliferation rate of the cells with or without overexpression of $\mathrm{Gfi}-\mathrm{I}$ at the time points indicated. $* P<0.05$ and $* * P<0.0 \mathrm{I}$. (C) Effect of Gfi-I overexpression on colony formation was measured in Hela and $\mathrm{SiHa}$ cells. The cells were seeded into six-well plates and cultured for 10 days, followed by crystal violet staining. The colony count is shown below. $* P<0.05$, $* * P<0.0 \mathrm{I}$, and $* * * P<0.00 I$. (D) Transwell assay was performed with the cells to measure the migration rate. Three independent experiments yielded similar results. (E) Cells were transfected with Gfi-I for $48 \mathrm{~h}$, and these cells were used to perform cell cycle analysis. Statistical significance was calculated using the Student's t-tests when only two groups were compared. $* P<0.05$, $* * P<0.01$, and $* * * P<0.001$.

suppressor genes destroy the equilibrium state of cell growth. Cells lose normal control and, therefore, develop tumors or promote tumor progression. ${ }^{22}$

The Gfi-1 gene encodes a $55 \mathrm{kDa}$ nuclear protein that contains six $\mathrm{C}$-terminal $\mathrm{C}_{2} \mathrm{H}_{2}$ zinc finger domains with
DNA-binding properties and an important transcriptional repressor essential to the 20 -amino acid barrier of the SNAG terminal domain. Gfi-1 inhibits T-cell activation by phase G1 arrest. $^{7}$ Gfi-1 also inhibits the regulation of apoptosis in a variety of apoptotic cells. ${ }^{23}$ Meanwhile Gfi- 1 counts 
A

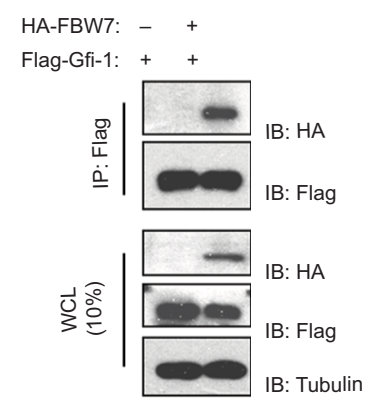

B

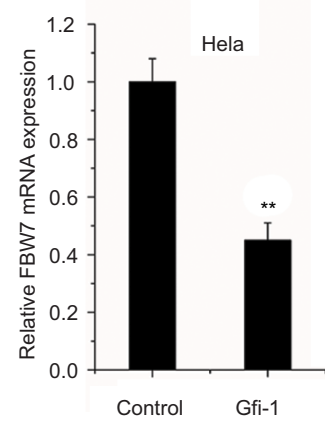

E

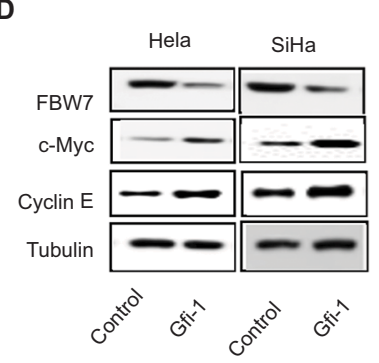

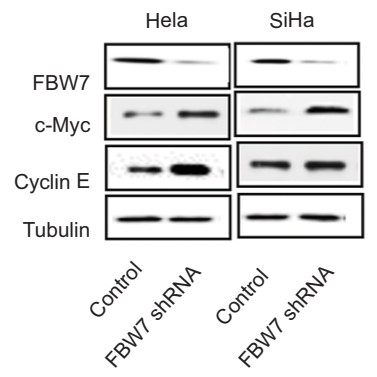

C

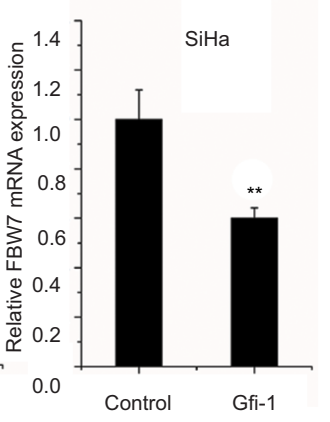

$\mathbf{F}$

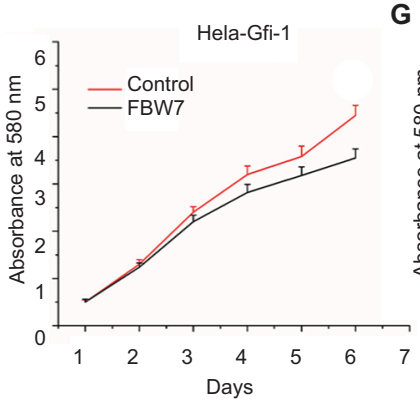

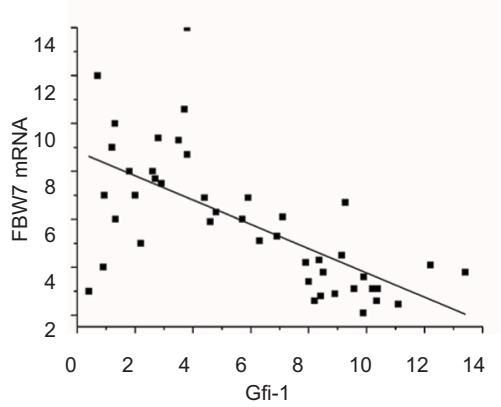

G

Figure $3 \mathrm{Gfi}-\mathrm{I}$ target of FBW7 exerts its function by suppressing FBW7 expression.

Notes: (A) 293T cells were transfected with Gfi-I either alone or together with FBW7. Whole-cell extracts were subjected to precipitation using HA antibody and examined for the indicated proteins by Western blot analysis. (B) Real-time quantitative PCR was used to detect the expression of FBW7 in two cervical cancer cell lines. (C) In cervical cancer, the negative correlation between Gfi-I and FBW7 mRNA was observed. Data are represented as the mean \pm SD, $n=3$. **P $<0.01$. (D and E) Expression of FBW7 gene and expression of c-Myc and cyclin E in cervical cancer cells were decreased by Gfi-I overexpression. The reduction in FBW7 expression increased the expression of c-Myc and cyclin $E$ in cervical cancer cells. ( $\mathbf{F}$ and $\mathbf{G})$ Growth of cervical cancer cells and detection by MTT.

Abbreviation: PCR, polymerase chain reaction.

A

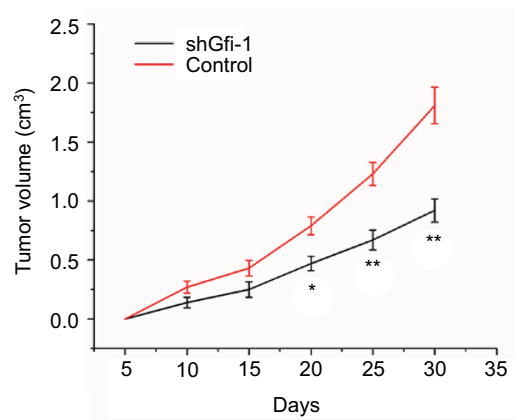

D

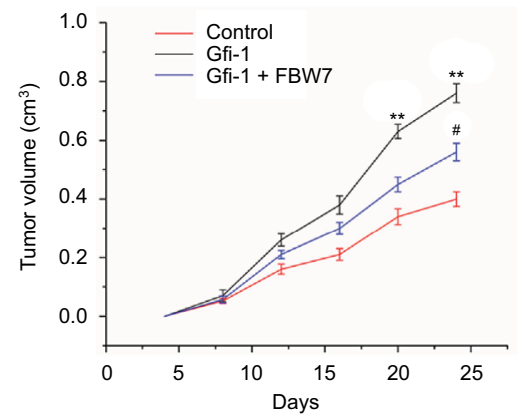

B

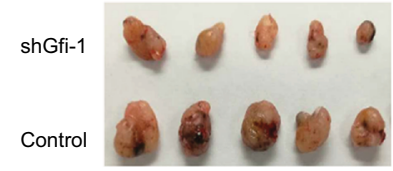

$\mathbf{E}$

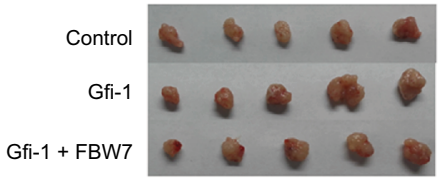

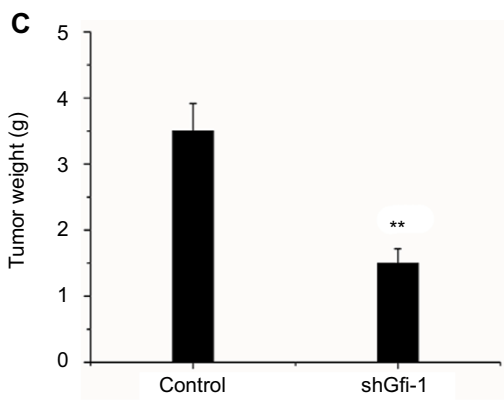

$\mathbf{F}$

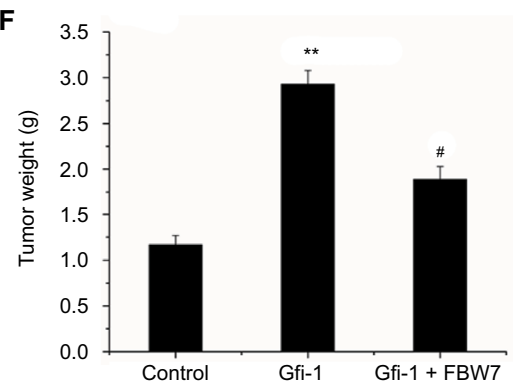

Figure 4 FBW7 blocks the Gfi-I-induced proliferation of Hela cells in vivo.

Notes: (A and B) Tumor volume was calculated every 5 days after the injection of shGfi-I Hela cells. Error bars indicate mean \pm SD. (C) Tumor weights are represented as mean of tumor weights $\pm S D$. ${ }^{* P}<0.05$, $* P<0.0$ I vs control. ( $\mathbf{D}$ and $\left.\mathbf{E}\right)$ Tumor volume was calculated every 5 days after the injection of Gfi-I overexpression cells and Gfi-I and FBW7 overexpression cells. (F) Tumor weights are represented as mean of tumor weights $\pm S D$. ${ }^{*} P<0.05$, ${ }^{* * P}<0.01$ vs control group, and ${ }^{\#} P<0.05$ vs $G f i-I$ overexpression group. 
E2F5, E2F6, Ets2, cMyc, and P21/WAF among its targets in myeloid cell lines. ${ }^{24}$ Although Gfi-1 has been implicated in the pathogenesis of lymphomas, little is known about its role in cervical cancer cells. Considering the present results, we hypothesized that high Gfi-1 expression promotes the invasion and metastasis of cervical cancer. High expression of Gfi-1 is associated with risk factors for cervical cancer, such as poor grade, invasion, and metastasis.

As a tumor suppressor gene, recent studies have found that FBW7 is closely related to aberrant expression in a variety of human malignancies and is related to the prognosis of malignant tumors. FBW7 has anti-tumor effect and promotes oncogene ubiquitination and degradation, such as c-Myc, Mcl-1, cyclin E, and c-Jun, and metabolism and regulation of cell proliferation, cell differentiation, apoptosis, and other cellular processes. ${ }^{25,26}$ In this study, we showed that Gfi-1 directly bound the FBW7 and Gfi-1 knockdown enhanced FBW7 expression. In addition, the expression of Gfi-1 and FBW7 gene mRNA in cervical cancer tissues was negatively correlated.

\section{Conclusion}

Our results indicate that high expression of Gfi-1 is a common event in cervical cancer. Increasing Gfi-1 expression promotes cell proliferation and inhibits FBW7 ubiquitin ligase expression. This suggests that a subset of Gfi-1 was a potential therapeutic target and biomarker.

\section{Acknowledgment}

This work was supported by grants from National Natural Science Fund for Distinguished Young Scholars (no 81302274). ZL is the first author and corresponding author.

\section{Disclosure}

The authors report no conflicts of interest in this work.

\section{References}

1. Jemal A, Bray F, Center MM, Ferlay J, Ward E, Forman D. Global cancer statistics. CA Cancer J Clin. 2011;61(2):69-90.

2. Ferlay J, Shin HR, Bray F, Forman D, Mathers C, Parkin DM. Estimates of worldwide burden of cancer in 2008: GLOBOCAN 2008. Int $J$ Cancer. 2010;127(12):2893-2917.

3. Williams-Brennan L, Gastaldo D, Cole DC, Paszat L. Social determinants of health associated with cervical cancer screening among women living in developing countries: a scoping review. Arch Gynecol Obstet. 2012;286(6): 1487-1505.

4. Becker S. A historic and scientific review of breast cancer: the next global healthcare challenge. Int J Gynaecol Obstet. 2015;131(suppl 1): S36-S39.

5. Duenas-Gonzalez A, Campbell S. Global strategies for the treatment of early-stage and advanced cervical cancer. Curr Opin Obstet Gynecol. 2016;28(1):11-17.
6. Cho U, Kim HM, Park HS, Kwon OJ, Lee A, Jeong SW. Nuclear expression of GS28 protein: a novel biomarker that predicts worse prognosis in cervical cancers. PLoS One. 2016;11(9):e0162623.

7. Zweidler-Mckay PA, Grimes HL, Flubacher MM, Tsichlis PN. Gfi-1 encodes a nuclear zinc finger protein that binds DNA and functions as a transcriptional repressor. Mol Cell Biol. 1996;16(8):4024-4034.

8. Zeng H, Yucel R, Kosan C, Klein-Hitpass L, Moroy T. Transcription factor Gfi-1 regulates self-renewal and engraftment of hematopoietic stem cells. EMBO J. 2004;23(20):4116-4125.

9. Moroy T, Khandanpour C. Growth factor independence 1 (Gfi-1) as a regulator of lymphocyte development and activation. Semin Immunol. 2011;23(5):368-378.

10. van Lohuizen M, Verbeek S, Scheijen B, Wientjens E, van der Gulden $\mathrm{H}$, Berns A. Identification of cooperating oncogenes in E mu-myc transgenic mice by provirus tagging. Cell. 1991;65(5):737-752.

11. Gilks CB, Bear SE, Grimes HL, Tsichlis PN. Progression of interleukin-2 (IL-2)-dependent rat $\mathrm{T}$ cell lymphoma lines to IL-2-independent growth following activation of a gene (Gfi-1) encoding a novel zinc finger protein. Mol Cell Biol. 1993;13(3):1759-1768.

12. Liu Q, Dong F. Gfi-1 inhibits the expression of eosinophil major basic protein (MBP) during G-CSF-induced neutrophilic differentiation. Int J Hematol. 2012;95(6):640-647.

13. Chalmin F, Mignot G, Bruchard M, et al. Stat3 and Gfi-1 transcription factors control Th17 cell immunosuppressive activity via the regulation of ectonucleotidase expression. Immunity. 2012;36(3):362-373.

14. Khandanpour C, Phelan JD, Vassen L, et al. Growth factor independence 1 antagonizes a 553 -induced DNA damage response pathway in lymphoblastic leukemia. Cancer Cell. 2013;23(2):200-214.

15. Suzuki J, Maruyama S, Tamauchi H, et al. Gfi-1, a transcriptional repressor, inhibits the induction of the $\mathrm{T}$ helper type 1 programme in activated CD4 T cells. Immunology. 2016;147(4):476-487.

16. Liu Q, Basu S, Qiu Y, Tang F, Dong F. A role of Miz-1 in Gfi1-mediated transcriptional repression of CDKN1A. Oncogene. 2010;29(19):2843-2852.

17. Welcker M, Clurman BE. FBW7 ubiquitin ligase: a tumour suppressor at the crossroads of cell division, growth and differentiation. Nat Rev Cancer. 2008;8(2):83-93.

18. Inuzuka H, Shaik S, Onoyama I, et al. SCF(FBW7) regulates cellular apoptosis by targeting MCL1 for ubiquitylation and destruction. Nature. 2011;471(7336):104-109.

19. Hong S, Laimins LA. The JAK-STAT transcriptional regulator, STAT-5, activates the ATM DNA damage pathway to induce HPV 31 genome amplification upon epithelial differentiation. PLoS Pathog. 2013;9(4):e1003295.

20. Xu Y, Yu J, Liu T, Meng F, Kong D, Lou G. Loss of FBXW7 is related to the susceptibility and poor prognosis of cervical squamous carcinoma. Biomarkers. 2016;21(4):379-385.

21. Shi GM, Ke AW, Zhou J, et al. CD151 modulates expression of matrix metalloproteinase 9 and promotes neoangiogenesis and progression of hepatocellular carcinoma. Hepatology. 2010;52(1):183-196.

22. McKenna NJ, O'Malley BW. Combinatorial control of gene expression by nuclear receptors and coregulators. Cell. 2002;108(4):465-474.

23. Grimes HL, Chan TO, Zweidler-McKay PA, Tong B, Tsichlis PN. The Gfi-1 proto-oncoprotein contains a novel transcriptional repressor domain, SNAG, and inhibits G1 arrest induced by interleukin-2 withdrawal. Mol Cell Biol. 1996;16(11):6263-6272.

24. Duan Z, Horwitz M. Targets of the transcriptional repressor oncoprotein Gfi-1. Proc Natl Acad Sci U S A. 2003;100(10):5932-5937.

25. Ibusuki M, Yamamoto Y, Shinriki S, Ando Y, Iwase H. Reduced expression of ubiquitin ligase FBXW7 mRNA is associated with poor prognosis in breast cancer patients. Cancer Sci. 2011;102(2): 439-445.

26. Iwatsuki M, Mimori K, Ishii H, et al. Loss of FBXW7, a cell cycle regulating gene, in colorectal cancer: clinical significance. Int J Cancer. 2010;126(8):1828-1837. 
Cancer Management and Research

\section{Publish your work in this journal}

Cancer Management and Research is an international, peer-reviewed open access journal focusing on cancer research and the optimal use of preventative and integrated treatment interventions to achieve improved outcomes, enhanced survival and quality of life for the cancer patient

The manuscript management system is completely online and includes

Submit your manuscript here: https://www.dovepress.com/cancer-management-and-research-journal

a very quick and fair peer-review system, which is all easy to use. Visit $\mathrm{http}: / / \mathrm{www}$.dovepress.com/testimonials.php to read real quotes from published authors. 